\title{
USE OF THE COLLABORATIVE OPTIMIZATION ARCHITECTURE FOR LAUNCH VEHICLE DESIGN
}

\author{
R.D. Braun* A.A. Moore ${ }^{\dagger}$ \\ Space Systems and Concepts Division \\ NASA Langley Research Center \\ Hampton, Virginia 23681
}

\author{
I.M. Kroo ${ }^{\ddagger}$ \\ Dept. of Aeronautics and Astronautics \\ Stanford University \\ Stanford, CA 94305
}

\begin{abstract}
$\underline{\text { Abstract }}$
Collaborative optimization is a new design architecture specifically created for large-scale distributed-analysis applications. In this approach, a problem is decomposed into a user-defined number of subspace optimization problems that are driven towards interdisciplinary compatibility and the appropriate solution by a system-level coordination process. This decentralized design strategy allows domain-specific issues to be accommodated by disciplinary analysts, while requiring interdisciplinary decisions to be reached by consensus. The present investigation focuses on application of the collaborative optimization architecture to the multidisciplinary design of a single-stage-to-orbit launch vehicle. Vehicle design, trajectory, and cost issues are directly modeled. Posed to suit the collaborative architecture, the design problem is characterized by 95 design variables and 16 constraints. Numerous collaborative solutions are obtained. Comparison of these solutions demonstrates the influence which an a priori ascent-abort criterion has on development cost. Similarly, objective-function selection is discussed, demonstrating the difference between minimum weight and minimum cost concepts. The operational advantages of the collaborative optimization architecture in a multidisciplinary design environment are also discussed.
\end{abstract}

\footnotetext{
* Aerospace Engineer, Senior member AIAA.

$\dagger$ Aerospace Engineer, Senior member AIAA.

$\ddagger$ Associate Professor, Senior member AIAA.

Copyright (C) 1996 by the American Institute of Aeronautics and Astronautics, Inc. No copyright is asserted in the United States under Title 17, U. S. Code. The U. S. Government has a royalty-free license to exercise all rights under the copyright claimed herein for Governmental purposes. All other rights are reserved by the copyright owner.
}

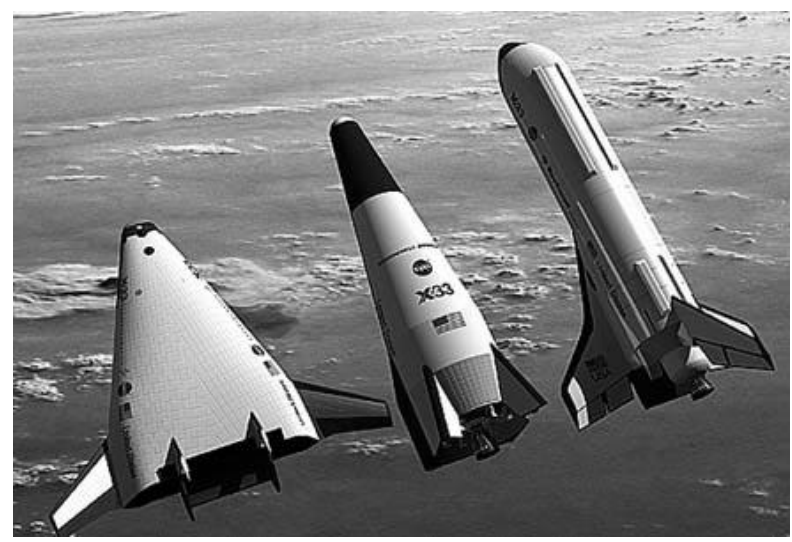

Figure 1. Industry-team Reusable Launch Vehicle concepts.

\section{Introduction}

For several years, NASA has been examining various Earth-to-orbit transportation options with the goal of reducing operating costs relative to the current U.S. launch fleet $[1,2,3,4]$. Many of these solutions have focused on fully reusable systems employing various levels of advanced technology $[5,6]$. Although a wide range of options have been examined, including single and two-stage systems using rocket and/or air-breathing propulsion, current emphasis has been placed on single-stage-to-orbit, rocket powered vehicles $[7,8,9,10]$. At present, several single-stage-to-orbit concepts are being studied by industry-led teams with support from NASA in the Reusable Launch Vehicle (RLV)/X-33 program $[10,11]$. Some preliminary design concepts from this study are illustrated in Fig. 1. The design of a single-stage-to-orbit launch vehicle is a complex, multidisciplinary process which is characterized by thousands of design variables and nonlinear constraints. A complete design requires analysis of aerodynamics, propulsion, weights and sizing, per- 
formance, heating, controls, operations, cost, and others [9]. While it is vital that each of these aspects be addressed at the conceptual level, the use of a design architecture that provides rapid performance of this multidisciplinary analysis while allowing the analyses to evolve as design maturity increases is equally imperative.

Enabled by the computational advances of the past few decades, a suite of multidisciplinary analysis and optimization architectures have emerged $[12$, 13]. Collaborative optimization is a new design architecture whose characteristics are well-suited to large-scale, distributed design [14]. The fundamental concept behind the development of this architecture is the belief that disciplinary experts should contribute to the design decision process while not having to fully address local changes imposed by other groups of the system. To facilitate this decentralized design approach, a problem is decomposed into subproblems along domain-specific boundaries. Through subspace optimization, each group is given control over its own set of local design variables and is charged with satisfying its own domain-specific constraints. Communication requirements are minimal since knowledge of the other groups' constraints or design variables is not required. The objective of each subproblem is to reach agreement with the other groups upon values of the interdisciplinary variables. A system-level optimizer is employed to orchestrate this interdisciplinary compatibility process while minimizing the overall objective. This decomposition strategy allows for the use of existing disciplinary analyses without major modification and is also well-suited to parallel execution across a network of heterogeneous computers [15].

Development of the collaborative optimization architecture is described in Refs. [14, 16, 17]. Comparison to other optimization architectures is made in Refs. [14, 17]. The architecture has been successfully used to solve several analytic test problems [14, 18], trajectory optimization problems [14, 16], and aircraft design problems $[15,19]$. The present investigation focuses on the application of this architecture to the multidisciplinary design of a single-stageto-orbit launch vehicle. Additional insight into this application is provided in Ref. [14].

\section{Nomenclature}

A nozzle area, $\mathrm{ft}^{2}$

c subspace constraints

F system-level objective

$\mathrm{F}_{z} \quad$ Wing normal force, $\mathrm{N}$

g system-level constraints

$\begin{array}{ll}\text { Isp } & \text { specific impulse, } \mathrm{s} \\ \mathrm{LH}_{2} & \text { liquid hydrogen } \\ \mathrm{LHC} & \text { liquid hydrocarbon } \\ \mathrm{LOX} & \text { liquid oxygen } \\ \mathrm{RP} & \text { kerosene } \\ \mathrm{Sref} & \text { wing area, } \mathrm{ft}^{2} \\ \mathrm{~T} & \text { thrust, } \mathrm{N} \\ \mathrm{T} / \mathrm{W} & \text { thrust-to-weight ratio } \\ \mathrm{x} & \text { interdisciplinary design vars. } \\ \bar{x} & \text { domain-specific design vars. } \\ \mathrm{y} & \text { interdisciplinary outputs } \\ \mathrm{z} & \text { system-level design vars. } \\ \alpha & \text { angle of attack, deg } \\ \Delta \mathrm{V} & \text { ideal velocity }\end{array}$

subscripts

$\begin{array}{ll}\text { c } & \text { computed } \\ \mathrm{e} & \text { exit } \\ \text { sl } & \text { sea-level } \\ \text { vac } & \text { vacuum }\end{array}$

superscripts

* subspace solution

** system-level solution

\section{Disciplinary Analyses}

In this analysis, design of a single-stage-to-orbit launch vehicle includes specification of the ascent trajectory, as well as determination of the subsystem weights and sizes, and assessment of the technology maturation and development costs. From the results of a previous study, an aerodynamically viable shape is modeled [20]. Propulsion system characteristics to be identified include the liftoff thrust-to-weight ratio, nozzle area ratio and fuel-to-oxidizer mixture ratios. Two mixture ratios require specification as the propulsion system is operated in different modes. From liftoff, liquid hydrogen and liquid hydrocarbon are both burned as fuel with liquid oxygen; while during a later portion of the ascent, only the hydrogen/oxygen mixture is used. The increased bulk density of such a dual-fuel concept has been shown to provide significant dry weight reductions [21]. The time to transition from mode 1 propulsion to mode 2 is also optimally determined. Development cost is the minimization variable which, in the present analysis, is defined to include the vehicle hardware design and development cost incurred under a full-scale development program. Other cost elements such as program management, fees, reserves, and software are not included. 
Historically, preliminary launch vehicle design has not included cost issues within the design loop. Instead, the pertinent engineering analyses are integrated and optimized to find either a minimum dry weight or minimum gross liftoff weight vehicle. A costing analysis is subsequently provided and, time permitting, some iteration may be performed. Because the goal of the RLV program is to provide technology development and demonstration of a lowcost, reliable, space transportation system [11], this traditional approach is no longer appropriate. In this case, it is imperative to consider cost issues at the preliminary design stage $[22,23]$. Hence, in this investigation, the impact of including cost within the preliminary design process is discussed for a representative, single-stage-to-orbit concept.

The vehicle is sized to deliver and return a 25,000 lb payload to the Space Station following launch from the Eastern Test Range at the Kennedy Space Center. For this analysis, the Space Station is assumed to be in a $220 \mathrm{n}$.mi. altitude orbit with a 51.6 degree inclination. The single-stage-to-orbit vehicle is flown into a $50 \times 100$ n.mi. altitude orbit with the correct inclination; on-board propellant is used to transfer to and circularize at 220 n.mi. Burnout constraints on altitude, flight-path angle, and inclination are enforced as are maximum inflight normal force, angle of attack, pitch rate, and dynamic pressure limits. An extension limit is placed on the dual-position rocket nozzle. In addition, technology maturation costs are constrained along a representative funding profile [24].

\section{Propulsion Analysis}

Propulsion system parametrics were supplied by Pratt and Whitney based on characteristics of the Russian RD-701 dual-fuel engine [21]. This system can burn either a hydrogen/kerosene mixture (mode 1 ) or pure hydrogen (mode 2) as fuel. During mode 1 , hydrogen is included in the fuel mixture to provide nozzle cooling and increased Isp. To further enhance performance, the dual-fuel engine is fitted with a dual-position nozzle. After a regression analysis, this parametric data can be used as shown in Fig. 2. Given the two nozzle area ratios and fuelto-oxidizer mixture ratios, numerous engine parameters are computed. These parameters include sealevel engine thrust-to-weight, specific impulse, and maximum allowable thrust (required inputs to the weights and sizing analysis) as well as vacuum thrust and nozzle exit area (required trajectory inputs). A $2: 1$ exit area limit is placed on the allowable extension of the dual-position nozzle to accommodate packaging of multiple engines on the vehicle base.

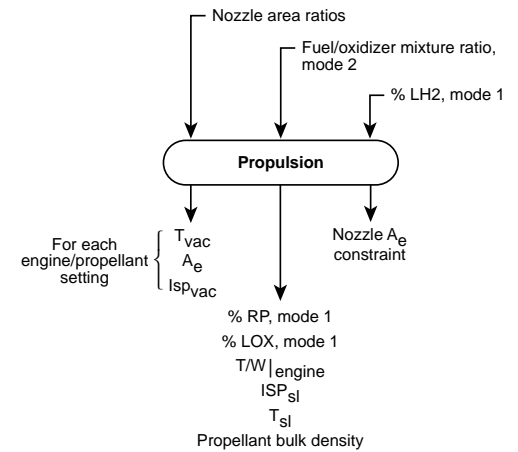

Figure 2. Propulsion analysis inputs and outputs.

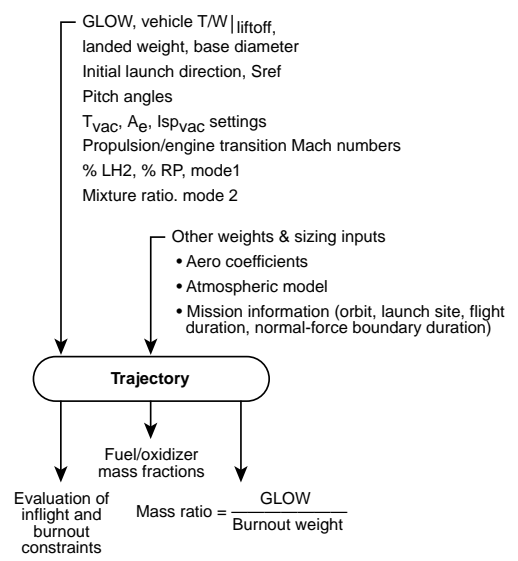

Figure 3. Trajectory analysis inputs and outputs.

\section{Trajectory Analysis}

To analyze the ascent flight-path, a three degree-of-freedom trajectory analysis is performed with the Program to Optimize Simulated Trajectories (POST) [25]. Within POST, the equations of motion are numerically integrated from an initial to a terminal set of state conditions. Within the present investigation, the vehicle is treated as a point-mass, Earth rotation and oblateness are modeled, and the 1976 standard atmosphere (no winds) is used. As shown in Fig. 3, the required set of trajectory inputs includes vehicle (e.g., gross liftoff weight, liftoff thrust-to-weight ratio, and aerodynamic coefficients and reference area) as well as trajectory parameters (pitch angle history, launch azimuth, and the propulsion-system transition Mach number). This domain-specific analysis is responsible for evaluation of the inflight and terminal constraints, computation of the vehicle mass ratio, and determination of the required fuel and oxidizer masses (weights and sizing inputs). Terminal constraints on altitude, velocity, and flight-path angle as well as maximum inflight dynamic pressure, angle of attack, pitch rate, and normal force (wing sizing constraint based on landed weight) limits are enforced. 


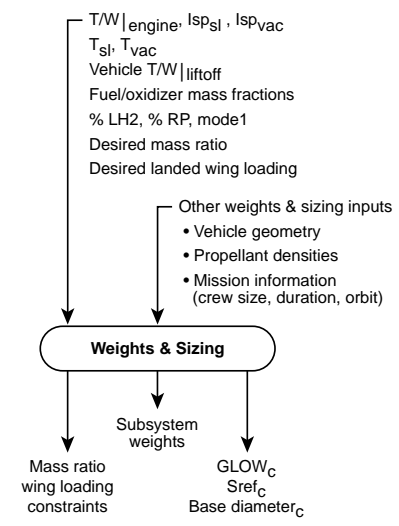

Figure 4. Weights and sizing analysis inputs and outputs.

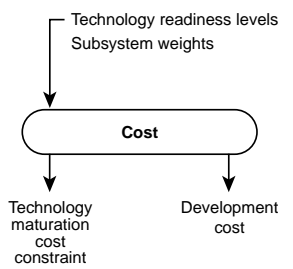

Figure 5. Cost analysis inputs and outputs.

Weights and Sizing Analysis

The Configuration Sizing program (CONSIZ) developed at NASA Langley Research Center is used to size the vehicle and determine subsystem weights. This sizing process is performed to meet vehicle mass ratio and landed wing loading constraints. As shown in Fig. 4, the liftoff thrust-to-weight, fuelto-oxidizer mixture ratio and fuel/oxidizer mass, as well as several propulsion system parameters are required input to CONSIZ. With the exception of the liftoff thrust-to-weight and the fuel-to-oxidizer mixture ratio, all of these inputs are computed by one of the other two disciplinary analyses. In addition to dry weight, CONSIZ computes the gross liftoff weight, reference aerodynamic surface area and landed weight (each of which is a required trajectory input). Numerous subsystem weights are also required as input to the cost analysis.

\section{Cost Analysis}

As depicted in Fig. 5, the cost model used in the present investigation consists of two sets of costestimating relationships. The first set, technology maturation costs, provide an estimate of the investment required to advance a given subsystem from its present technology readiness level to a higher technology readiness level within five years (the presumed beginning of the development cycle). As presented in Fig. 6, the NASA technology readiness scale was used as a measure of subsystem maturity. Data from Ref. [24] was used to compile these re-

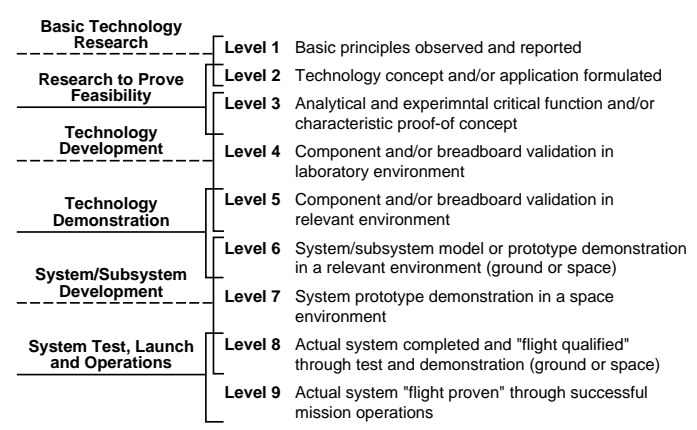

Figure 6. NASA technology readiness levels.

lations. For this estimation, the vehicle subsystems are partitioned into nine segments: avionics, structures, electromechanical actuation, electrical conversion and distribution, auxiliary propulsion, prime power, main propulsion, propellant tanks, and thermal protection. The total technology maturation cost is defined as the sum of the cost of maturing these nine elements.

The second set of nonlinear, vehicle-specific cost-estimating relationships were developed consistent with the subsystem breakdown structure of CONSIZ. These parametric relations predict subsystem development cost as function of weight, complexity, and technology readiness level. Additional relationships for subsystems which do not rely on advanced technology (e.g., landing gear) were included to obtain an estimate of the total vehicle development cost assuming a full-scale development program. The development cost relations were formulated through application of the Parametric Review of Information for Costing and EvaluationHardware (PRICE-H) multivariate model [26]. This model has been used to generate integrated cost and schedule estimates for numerous industry and government aerospace programs. The model provides a computational method for deriving cost estimates of electronic and mechanical hardware assemblies and systems.

\section{Interdisciplinary Coupling}

From Fig. 2 to Fig. 5, it is clear that solution of this problem requires an iterative approach as the each analysis each requires inputs which are computed by another discipline. For example, one must ensure that the reference aerodynamic surface area resulting from the vehicle sizing process (Sref $c_{c}$ of Fig. 4) is the same as the reference aerodynamic surface area used to compute the aerodynamic forces (Sref of Fig. 3). Interdisciplinary compatibility must also be achieved in regard to the gross liftoff weight, landed weight, base diameter, mass ratio, fuel/oxidizer mass, and each of the propulsion 


\begin{tabular}{|l|ccc|}
\hline \hline \multicolumn{1}{|c|}{$\begin{array}{c}\text { Interdisciplinary } \\
\text { Variable }\end{array}$} & Prop. & Traj. & $\begin{array}{c}\text { Weights } \\
\text { Cost }\end{array}$ \\
\hline Gross liftoff weight & & input & output \\
Aero ref. area & & input & output \\
Landed weight & & input & output \\
Base diameter & & input & output \\
Vehicle liftoff T/W & input & output \\
\% LH $H_{2}$ mode 1 & input & input \\
\% RP, mode 1 & output & input & input \\
Mixture ratio, mode 2 & input & input & \\
Vac. thrust, mode 1a & output & input & \\
Vac. Isp, mode 1a & output & input & \\
Vac. thrust, mode 1b & output & input & \\
Vac. Isp, mode 1b & output & input & \\
Vac. thrust, mode 2 & output & input & \\
Vac. Isp, mode 2 & output & input & \\
Noz. area, retracted (a) & output & input & \\
Noz. area, extended (b) & output & input & \\
Mass ratio & & output & input \\
LH mass fraction & & output & input \\
RP mass fraction & & output & input \\
Engine sea-level T/W & output & & input \\
Sea-level Isp, mode 1 & output & & input \\
Allowable thrust ratio & output & & input \\
\hline \hline & & & \\
\hline
\end{tabular}

Table 1. Interdisciplinary coupling present in launch vehicle design problem.

discipline outputs. The liftoff thrust-to-weight and fuel-to-oxidizer mixture ratios must be treated in a similar fashion since these parameters are input to more than one analysis. The interdisciplinary coupling structure of this launch vehicle design problem is shown in Table 1. Here, the input/output structure of the propulsion (Prop.), trajectory (Traj.), and weights, sizing, and cost subspaces is depicted. In this table, the weights, sizing, and cost analyses are grouped together since they are integrated within the same subspace in the collaborative architecture solutions.

\section{Potential Optimization Architectures}

Numerous optimization architectures have been proposed for launch vehicle design. In this section, some of the potential solution strategies are discussed. The design problem, as posed within the collaborative optimization architecture, is then presented.

The disciplinary tools used in this analysis were originally developed as stand-alone programs, each operated by a disciplinary expert. To obtain a credible vehicle, a design team was required to manually iterate among these disciplinary analyses. In many cases, "optimization" was performed through trade- studies in which the parameters were varied one at a time [27, 28].

Improvement over this one-variable-at-a-time approach has been achieved using response surface methods (RSM) [9, 20, 21, 29, 30]. In a RSM strategy, feasible designs are computed at numerous points in the design space and a surface is fit to these points. Optimization is then performed on this approximate representation of the design space. Although use of RSM is a significant improvement over one-variable-at-a-time trade studies, the method suffers from several drawbacks including the requirement to produce numerous feasible design candidates and the limitation to problems characterized by a relatively small number of variables. For larger applications, as of interest in this work, the use of RSM is not a viable option.

Numerous gradient-based optimization architectures are possible for solution of this launch vehicle design problem. For example, use of system sensitivity analyses is presented in Ref. [31] for a similar problem (cost issues not addressed). Within the launch vehicle design community, the traditional multidisciplinary design optimization approach is to integrate the appropriate disciplinary analyses in a nonhierarchic iterative loop with a single, systemlevel optimizer $[32,33,34,35,36]$. In this standard optimization approach, interdisciplinary compatibility is enforced through some form of loop convergence criteria. As a result, numerous analysis evaluations are required to produce a single design candidate. An all-at-once architecture has also been proposed for launch vehicle design $[23,36]$. In this approach, the iterative loop of the standard approach is removed through the use of auxiliary variables and compatibility constraints $[15,37]$. As demonstrated in Ref. [36], use of a sequential-analysis, all-at-once optimization architecture may significantly reduce the required number of function evaluations. In a subsequent section, the standard and all-at-once solution strategies presented in Ref. [36] are compared to the use of collaborative optimization.

\section{Collaborative Optimization}

Figure 7 shows the launch vehicle design problem as posed to suit the collaborative optimization architecture. Here, the design is decomposed into three subspace coordinated by a system-level optimization procedure. Note that the weights, sizing, and cost issues are all accommodated within a single subspace. This decomposition strategy was selected because the weight and cost models are tightly coupled through numerous subsystem weights. For example, in the present model, development cost is a 


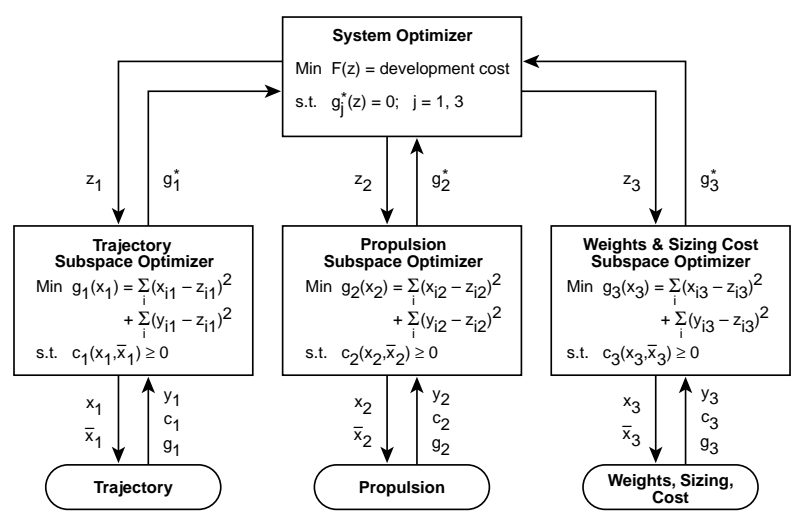

Figure 7. Collaborative optimization architecture for launch vehicle design.

\begin{tabular}{|l|cc|}
\hline \hline \multicolumn{1}{|c|}{$\begin{array}{c}\text { Optimization } \\
\text { Level }\end{array}$} & $\begin{array}{c}\text { Design } \\
\text { Var. }\end{array}$ & $\begin{array}{c}\text { Nonlinear } \\
\text { Const. }\end{array}$ \\
\hline System & 23 & 3 \\
Trajectory subspace & 47 & 9 \\
Propulsion subspace & 4 & 1 \\
Weights, sizing, cost sub. & 21 & 3 \\
\hline \hline
\end{tabular}

Table 2. Collaborative optimization launch vehicle design characteristics.

function of 17 subsystem weights. Hence, in this case, analysis integration is useful in reducing the degree of interdisciplinary coupling.

Posed to suit the collaborative architecture, the problem is characterized by 95 design variables and 16 constraints. These design variables and constraints are partitioned among the system-level and subspaces as listed in Table 2. Further insight into the subspace and system-level problems is provided in Ref. [14].

Numerous collaborative solutions were obtained for this launch vehicle design problem. In each case, the subspace bounds for the interdisciplinary variables were not selected consistently. For example, in the trajectory subspace, the vehicle liftoff $\mathrm{T} / \mathrm{W}$ was required to be between 1.0 and 1.3 ; whereas, a range of 1.0-1.5 was placed on this interdisciplinary input within the weights, sizing, and cost subspace. Similarly, the percentage of $\mathrm{LH}_{2}$ in mode 1 had bounds of $0-15,3-20$, and $3-15$ within the various subspaces. These bound inconsistencies were included to simulate the architecture's application in a team environment where the various disciplinary specialists are each responsible for a particular domain-specific subproblem.

Prior to optimization, the subspace and systemlevel problems were scaled such that the design variables, constraints, and objective function were of

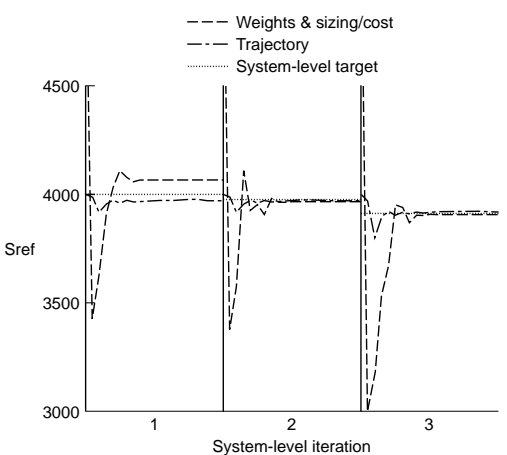

Figure 8. Interdisciplinary negotiation within the collaborative optimization architecture.

order one. However, in this investigation, no attempt was made to provide a twice-continuously differentiable model. A majority of the sources which contribute nonsmoothness are within the trajectory subspace-the ascent pitch profile which is modeled by discrete control points connected by linear segments as well as tabular linear interpolation of the aerodynamic and atmospheric properties (1976 standard model).

As an example of the collaborative convergence process, Fig. 8 depicts the interdisciplinary negotiation that occurs between the trajectory subspace, weights-sizing-cost subspace, and system-level on the appropriate value of the wing aerodynamic reference area (Sref). This figure shows the domainspecific convergence histories for 3 system-level iterations. In the first iteration, the system-level proposes an Sref value of $4000 \mathrm{ft}^{2}$. After meeting all of its domain-specific constraints, the trajectory subspace returns with a request to change this value to $3950 \mathrm{ft}^{2}$. Similarly, the weights-sizing-cost subspace returns with hopes of increasing this value to $4100 \mathrm{ft}^{2}$. Based on gradient information provided at the solution of each subspace, a system-level step is taken which alters the system-level value Sref to $3955 \mathrm{ft}^{2}$ (and the value of the other 22 systemlevel variables). In this case, the subspaces are able to remain disciplinary constraint feasible while providing better agreement on this value of Sref. In the third iteration, the value of Sref is reduced even further with consent from the two domainspecific analyses. Through repeated collaboration, the system-level optimizer orchestrates the interdisciplinary compatibility process. Note that in Fig. 8, cold-start subspaces were used for illustrative purposes. Hence, within each system-level iteration, subspace optimization was initialized from a fixed, domain-specific point (an Sref of $4000 \mathrm{ft}^{2}$ in the trajectory subspace and an Sref of $5000 \mathrm{ft}^{2}$ in the 


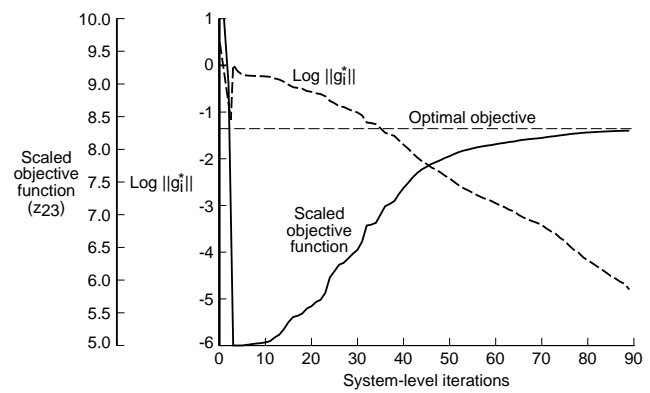

Figure 9. Collaborative optimization system-level convergence profile. Minimum development cost concept.

weights-sizing-cost subspace). In the results which follow, subspace optimization is initialized from the prior solution (warm-start) to reduce the computational requirements of repeated subspace optimization.

\section{Application of the Collaborative Optimization Architecture}

In this section, optimal solutions of several versions of the basic launch vehicle design problem are presented. Each case was obtained through application of the collaborative optimization architecture. To obtain these solutions, the refined architecture of Ref. [14] was used. This implementation includes use of the linear system-level objective refinement as well as the warm-start and slack-variable subspace refinements presented in Ref. [14]. The system-level Jacobian is obtained from the use of post-optimality information estimated at subspace solutions as discussed in Refs. [14, 38]. The sequential quadratic programming algorithm, NPSOL [39], was used to provide system-level and subspace optimization. CPU times quoted are based on use of a Silicon Graphics Challenge L machine outfitted with R8000, $90 \mathrm{MHz}$ processors.

\section{Minimum Development Cost Concept}

The collaborative optimization convergence history for this solution is presented in Fig. 9. The 89 system-level iterations shown required 181 subspace optimizations of each of the three domain-specific analyses. The solution obtained compares favorably (within $0.1 \%$ in the system-level objective function) with the solution presented in Ref. [23] where an all-at-once optimization strategy was used. As discussed in Ref. [14], this convergence profile, where the system-level objective initially drops and then approaches the solution from below as system-level feasibility is achieved, is similar to collaborative solutions obtained for other problems.

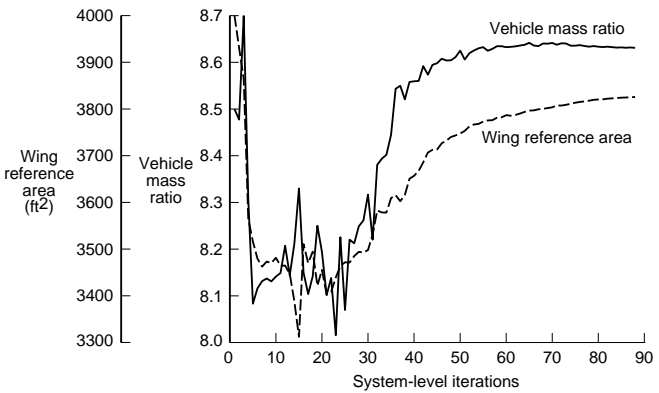

Figure 10. Collaborative optimization convergence history: system-level values of vehicle mass ratio and wing aerodynamic reference area.

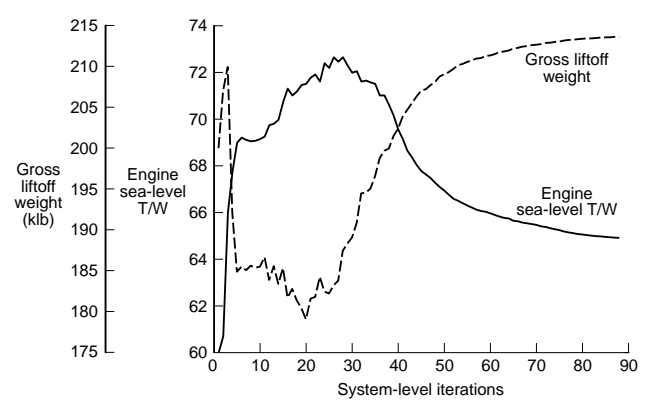

Figure 11. Collaborative optimization convergence history: system-level values of main engine sea-level $\mathrm{T} / \mathrm{W}$ and vehicle gross liftoff weight.

For the solution shown in Fig. 9, 181 sets of subspace optimization were performed. Initialized from a fixed initial guess (cold-start), a call to the subspaces requires approximately 1-3 hours. At this rate, a cold-start solution requires approximately 1-3 weeks. Using warm-start subspaces (restarting from the previous domain-specific solution with knowledge of the prior optimum active set, Lagrange multipliers, and Hessian of the Lagrangian), the solution presented in Fig. 9 required approximately 4.5 days of computer time. Hence, warm-starting the subspaces provides a dramatic advantage. This level of efficiency gain is possible because the subspaces are tasked with solving a related sequence of subproblems.

Figures 10, 11, and 12 demonstrate the convergence behavior of six of the interdisciplinary variables. These figures depict the system-level values of the vehicle mass ratio, wing aerodynamic reference area, main engine sea-level $\mathrm{T} / \mathrm{W}$, vehicle gross liftoff weight, vehicle liftoff $\mathrm{T} / \mathrm{W}$, and the mode 2 mixture ratio. As shown, the system-level variables tend to exhibit either the same behavior as the system-level objective or a more damped oscillation about the optimal value.

The flight path portion of this optimal solution is illustrated in Figs. 13 and 14. The ascent begins 


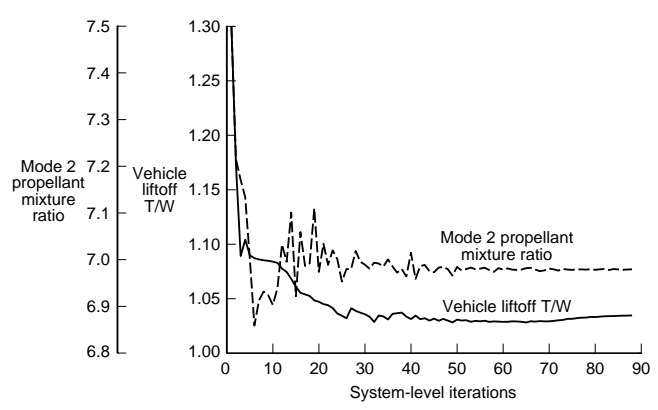

Figure 12. Collaborative optimization convergence history: system-level values of vehicle liftoff $\mathrm{T} / \mathrm{W}$ and oxidizer/fuel mixture ratio during mode 2 propulsion.

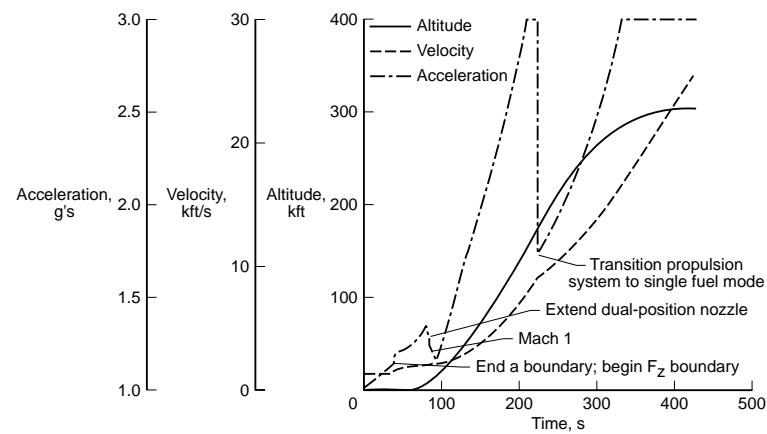

Figure 13. Optimal flight profile (altitude, velocity, and acceleration).

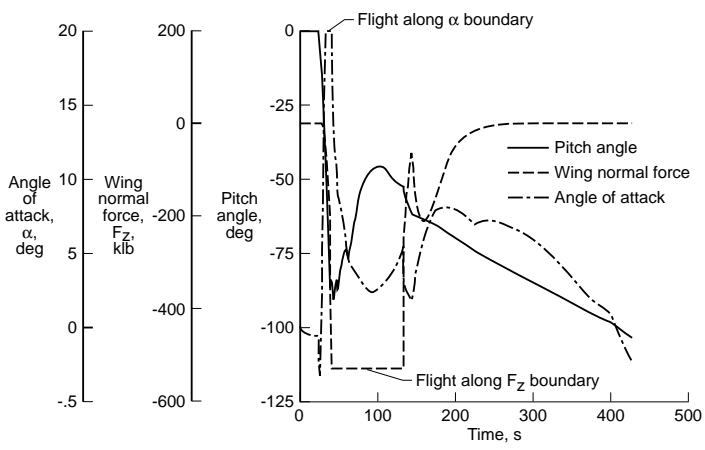

Figure 14. Optimal pitch-angle and inflight constraint profiles.
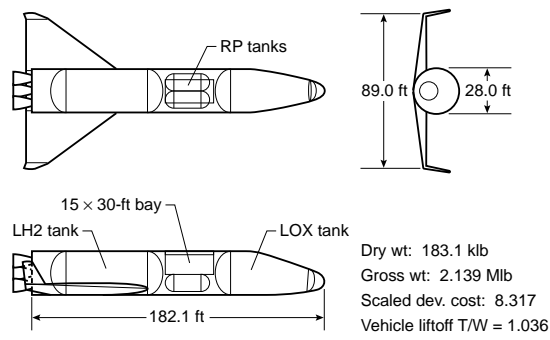

Figure 15. Minimum development-cost vehicle description, no low-altitude ascent abort constraint.

with a $400 \mathrm{ft}$ vertical rise to clear the launch facility. Because the vehicle is characterized by a low liftoff $\mathrm{T} / \mathrm{W}(1.036)$, this rise takes approximately 20 $\mathrm{s}$. This vertical flight segment is followed by a maximum pitch-rate segment in which the vehicle tries to attain its maximum-lift orientation. The pitchrate is limited to $5 \mathrm{deg} / \mathrm{s}$ to reflect control issues which are not modeled in this analysis. As shown in Fig. 14, during this segment of flight, angle of attack $(\alpha)$ increases until it reaches the allowed maximum of 20 deg. Flying at this $\alpha$, the normal force builds until a limiting load is reached. The vehicle rides this normal-force boundary through peak dynamic pressure which for the optimum flight path is about 990 psf. Hence, at this solution, the dynamic pressure limit of $1000 \mathrm{psf}$ is not active. During this phase of flight at approximately $10 \mathrm{kft}$, the back pressure losses are low enough that the dual-position nozzle is extended to gain propulsive efficiency. As the nozzle extension is performed, the vehicle acceleration initially decreases as a result of flight through the transonic regime. At about $50 \mathrm{kft}$, as the dynamicpressure decreases, the vehicle comes off the normalforce boundary but continues to accelerate towards $3 \mathrm{~g}$ 's. The vehicle reaches $3 \mathrm{~g}$ 's while in the dualfuel propulsive mode and uses engine throttling to maintain this level of acceleration. In this analysis, transition of all seven engines from a dual-fuel to single-fuel mode is performed instantaneously. This instantaneous change in thrust results in the large decrease in acceleration shown in Fig. 13 at roughly $225 \mathrm{sec}$ and an altitude of about $175 \mathrm{kft}$. Operating in a single-fuel mode $\left(\mathrm{LH}_{2}\right)$, the vehicle accelerates back to $3 \mathrm{~g}$ 's and holds this acceleration level until reaching orbit. Note that if the nozzle transition been performed sequentially, a slightly better result could have been achieved.

This minimum development cost configuration is sketched in Fig. 15. As shown in this figure, the vehicle is characterized by a dry weight of $1.831 \mathrm{x}$ $10^{5} \mathrm{lb}$, a gross liftoff weight of $2.139 \times 10^{6} \mathrm{lb}$ and a length of $181.6 \mathrm{ft}$. Note that the optimum vehicle 


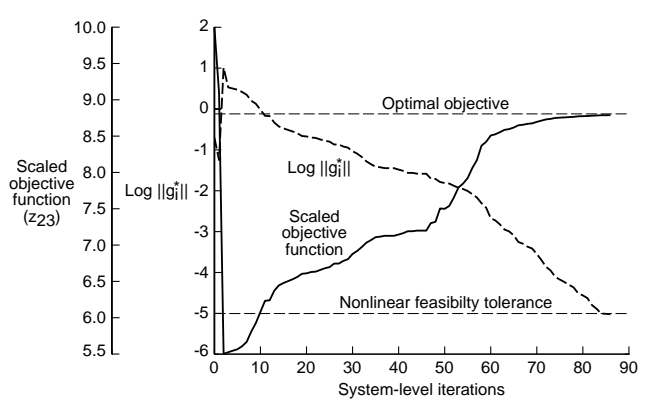

Figure 16. Collaborative optimization system-level convergence profile. Minimum development cost concept (vehicle liftoff $\mathrm{T} / \mathrm{W} \geq 1.2$ ).

$\mathrm{T} / \mathrm{W}$ is 1.036 at liftoff. This $\mathrm{T} / \mathrm{W}$ level, which is significantly lower than the 1.2 value typically observed, is the subject of the following section.

\section{How Many Engines?}

With the system-level vehicle liftoff $\mathrm{T} / \mathrm{W}$ lower bound increased to 1.2 , the collaborative optimization architecture was re-run, giving the systemlevel performance profiles shown in Fig. 16. Here, 86 system-level iterations are required to obtain system-level convergence. Comparison of the solutions presented in Figs. 9 and 16 demonstrates that requiring a vehicle liftoff $\mathrm{T} / \mathrm{W} \geq 1.2$ induces a $4.5 \%$ increase in development cost.

Generally, a lower bound on the vehicle liftoff $\mathrm{T} / \mathrm{W}$ is included at the preliminary design stage to provide sufficient engine-out capability during the first moments of ascent (vehicle $\mathrm{T} / \mathrm{W}$ increases with time). In this case, 1.2 was chosen because the vehicle was designed with 7 engines. Hence, with loss of a single engine at liftoff, the vehicle would still have propulsive control authority-the $\mathrm{T} / \mathrm{W}$ would become 1.03 [40]. Inclusion of this engine-out capability is the primary reason that many launch vehicle design concepts have a liftoff $\mathrm{T} / \mathrm{W}$ of 1.2 (e.g., Refs. $[7,9,20,21,31,36])$. Unfortunately, this increased flexibility does not come without a price, as the minimum development cost concept would prefer a lower liftoff $\mathrm{T} / \mathrm{W}$.

To further complicate this issue, the number of engines is a significant driver on vehicle cost and reliability. As the number of engines increases, flight reliability concerns also increase. To maintain a certain level of system reliability, e.g., $99 \%$, each engine in a 7 engine cluster must have a reliability of $99.86 \%$. With just 3 engines, this individual engine reliability is reduced to $99.66 \%$. Since reliability is a significant cost driver $[1,6]$, low-cost concepts such as those envisioned within the RLV program may not have the luxury of including this engine-out abort constraint. For example, with 3 engines, incorporation of a liftoff engine-out capability induces a severe penalty since the lower bound on the allowable liftoff $\mathrm{T} / \mathrm{W}$ would be increased to 1.5. In this case, this liftoff engine-out requirement may have to be discarded. Although this is the philosophy selected in the single-stage-to-orbit designs of the present investigation, it is clear that this issue requires further study.

\section{Objective Selection in Launch Vehicle Design}

The concept of a "best" or optimal configuration has different meanings in different design groups. In launch vehicle design, minimum weight concepts have traditionally been sought. In some cases, liftoff gross weight is selected as the minimization variable $[27,28]$. However, with the realization that propellant is relatively inexpensive, a majority of the recent concepts have been design to minimum dry weight (e.g., Refs. [7, 9, 20, 21, 31, 36]). Often, these minimum dry weight studies include a claim such as, "Since vehicle development costs tend to vary as a function of dry weight, this minimum dry weight vehicle may be considered a minimum development cost concept". However, as demonstrated in this section, such an assertion is not rigorously true even when a weight-based cost model is used.

Another objective function which has been suggested within the launch vehicle design community is minimum $\Delta \mathrm{V}$. This objective is sometimes chosen based on application of the "rocket equation". However, as shown in this section, minimum $\Delta \mathrm{V}$ concepts are vastly different from either minimum development cost or minimum weight designs. In this case, it is the use of analysis approximation (i.e., use of the rocket equation instead of a higher-fidelity trajectory simulation program) which leads to such a dramatically different concept.

Distinctions among these four different objectives (gross weight, dry weight, development cost, and $\Delta V$ ) are examined through application of the collaborative architecture. In each of these designs, the vehicle liftoff $\mathrm{T} / \mathrm{W}$ is allowed to vary in the range 1.0-1.5. The minimum development cost solution, described in the previous section, is used to normalize the other optimal results.

Design characteristics of these four optimal concepts are listed in Table 3 . As shown by the fourth column of this table, at the vehicle level, the minimum $\Delta V$ concept stands apart from the other 3 designs. This system is over $50 \%$ more expensive than the minimum development cost concept and more than $25 \%$ heavier than the minimum dry weight case (while yielding only a $4-6 \%$ improvement in $\Delta V$ ). 


\begin{tabular}{|c|c|c|c|c|}
\hline \multirow{3}{*}{$\begin{array}{c}\text { Normalized } \\
\text { Design } \\
\text { Characteristics }\end{array}$} & \multicolumn{4}{|c|}{ Objective Function } \\
\hline & Dev. & Dry & Liftoff & $\Delta V$ \\
\hline & Cost & Weight & Weight & \\
\hline Dev. cost & 1.0 & 1.001 & 1.040 & 1.510 \\
\hline Dry weight & 1.0 & 0.987 & 1.035 & 1.262 \\
\hline Liftoff weight & 1.0 & 1.0 & 0.9 & 1.196 \\
\hline$\Delta \mathrm{V}$ & 1.0 & 0.999 & 0.9 & 0.944 \\
\hline Body length & 1.0 & 0.993 & 1.030 & 1.052 \\
\hline Wing area & 1.0 & 0.993 & 1.043 & 1.239 \\
\hline Liftoff $\mathrm{T} / \mathrm{W}$ & 1.0 & 1.004 & 1.082 & 1.448 \\
\hline Mass Ratio & 1.0 & 1.008 & 0.893 & 0.959 \\
\hline
\end{tabular}

Table 3. Optimal launch vehicle comparison.

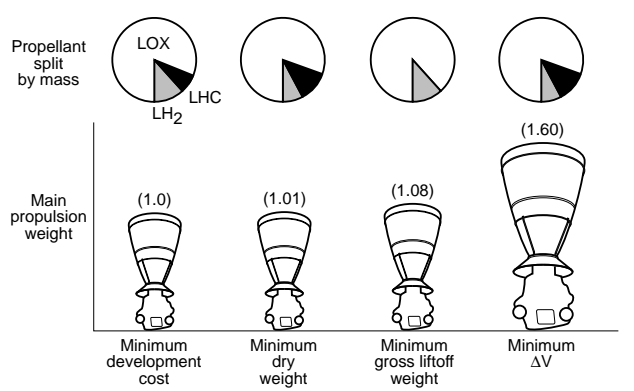

Figure 17. Optimal launch vehicle comparisonmain propulsion system.

Furthermore, the vehicle's wing area is more than $20 \%$ larger than each of the other wings. With the exception of this concept, the other three concepts share a top-level similarity, the strongest correlation being between the minimum development cost and minimum dry weight configurations. However, even among these first three concepts, the maximum variance in dry weight is $3.5 \%$, the maximum variance in development cost is $4.0 \%$, and the maximum variance in gross liftoff weight is $6.7 \%$.

The optimal liftoff $\mathrm{T} / \mathrm{W}$ and mass ratio for each of the four concepts is also listed in Table 3. From the liftoff $\mathrm{T} / \mathrm{W}$ comparison, it is clear why the minimum $\Delta V$ concept is so different. In this case, the optimization process attempts to minimize the ascent losses (e.g., gravitational, aerodynamic, nozzle backpressure, and thrust-vectoring) by achieving orbit as quickly as possible. As shown in Fig. 17, to accomplish this, the propulsion system size is greatly increased and a larger RP propellant mass fraction (LHC) is required. In this case, the vehicle liftoff $\mathrm{T} / \mathrm{W}$ is at its upper bound of 1.5 . Had this variable been allowed to increase even further, the vehicle would have continued to grow, reaching enormous proportions.
Figure 17 shows that subsystem differences are not limited to the minimum $\Delta V$ concept. For example, while a dual-fuel system is allowed, the minimum gross weight concept prefers $\mathrm{LH}_{2}$ as its sole fuel. In this case, the dense RP propellant is eliminated such that Isp is increased while the initial launch weight is reduced. This disregard for bulk density is in stark contrast to the minimum dry weight case which relies on bulk density to provide dry weight savings [21]. The goal of minimum gross liftoff weight also induces a $8.2 \%$ increase in vehicle liftoff $\mathrm{T} / \mathrm{W}$ and a $10.6 \%$ decrease in vehicle mass ratio (see Table 3 ) relative to the minimum development cost system.

Figure 17 also shows that the minimum development cost and minimum dry weight concepts are not equivalent. This finding is of even greater significance when one considers that the present analysis relies on a weight-based cost model. For example, relative to the minimum dry weight design, the minimum development cost concept uses $16 \%$ less RP (LHC). In this case, the minimum cost concept seeks to reduce propulsion system mass since this subsystem is characterized by both a high development cost per pound and technology maturation cost. In essence, the minimum development cost case may be thought of as a "weighted" minimum weight case, where all pounds are not created equal.

The results shown in this section demonstrate the importance of appropriate objective function selection. In particular, while minimum weight and minimum cost concepts may be similar, they are certainly not equivalent (even when a weight-based cost model is used). Differences at the subsystem level were shown to reflect the goal of the optimization process. Therefore, if one is truly interested in developing minimum cost concepts (the stated goal of the RLV program), cost considerations must be factored into the preliminary design process prior to optimization [22, 23].

\section{Operational Aspects of Architecture Selection}

In Ref. [14], the computational performance of the collaborative optimization architecture was shown to improve as the size of the domain-specific problems are increased without a significant increase in interdisciplinary coupling. Hence, using computational performance as a yardstick, application to large-scale, loosely-coupled problems was suggested. In a multidisciplinary design environment, computational performance is only one aspect of architecture selection. Often, in such a setting, analysis integration and communication requirements are much harder to efficiently resolve. In addition, system 


\begin{tabular}{|l|ccc|}
\hline \hline \multicolumn{1}{|c|}{$\begin{array}{c}\text { Optimization } \\
\text { Architecture }\end{array}$} & $\begin{array}{c}\text { Function } \\
\text { Evals }\end{array}$ & $\begin{array}{c}\text { Mod. } \\
\text { Time }\end{array}$ & $\begin{array}{c}\text { Comm. } \\
\text { Reqs. }\end{array}$ \\
\hline Std. approach, [36] & 10482 & $4 \mathrm{mo}$. & 66 \\
Collaborative opt. & $3125-24840$ & $1 \mathrm{mo}$. & 23 \\
All-at-once, [36] & 3182 & $3 \mathrm{mo}$. & 65 \\
\hline \hline
\end{tabular}

Table 4. Comparison of three multidisciplinary optimization strategies for launch vehicle design.

flexibility, analysis modularity, and resource management are also concerns. For example, if it requires a year to set up the appropriate multidisciplinary analysis system, run-time is certainly inconsequential. Furthermore, if a system is integrated in a manner such that it is difficult to modify or extend, it is not likely to have a long life within the design organization. As the number and fidelity of the domain-specific analyses increases, these operational concerns becomes more significant.

Based on the results of this paper and Ref. [36] (where cost issues were not addressed), Table 4 presents the author's experience solving a similar launch vehicle design problem with three optimization architectures. This table lists the function evaluations required to reach the solution from a common initial guess, the estimated analysis modification time (Mod. Time), and the interdisciplinary communication requirements (Comm. Reqs.) for each of the three optimization architectures. As a result of the variation in subspace problem sizes (see Table 2), the propulsion subspace required far fewer function evaluations than the trajectory subspace. This is the cause for the range of numbers listed in the function evaluation column of Table 4 for the collaborative architecture. This table shows that while, in its present form, the collaborative architecture is not computationally competitive with the all-atonce optimization architecture, it is generally competitive with the standard optimization approach. A similar conclusion was reached for the large-scale trajectory optimization problems of Ref. [14]. Because Table 4 lists function evaluations, the potential parallelization speedup inherent to the collaborative and the all-at-once approaches is not reflected.

Use of the collaborative architecture offers numerous other advantages. The largest of these is the estimated analysis modification time. Recall that this analysis is based on the modification of a set of previously stand-alone disciplinary programs. In such a case, the standard and the all-at-once approaches require a much higher degree of analysis modification. For the standard optimization approach, this time is spent providing integration of the disciplinary analyses. Since this integration was performed in an "engineering" sense, the resulting integrated system is not extremely flexible.

In the case of the all-at-once system, significant time was spent preparing the analyses for optimization. In a practical design environment, many disciplinary analyses are simply not set-up for efficient optimization ("design-oriented"). For example, in its original form, the trajectory analysis was not well-suited for function evaluation. Instead, trajectory evaluation was explicitly coupled with optimization. While the original analysis program was simply inserted into the collaborative architecture, significant time was spent providing a trajectory function evaluator for use with the other approaches.

Communications requirements of the collaborative architecture are also minimal (see Table 4), resulting solely from the interdisciplinary coupling inherent in the problem. In contrast, both the standard and all-at-once approaches required significantly more communication. In the standard approach, 36 variables were passed between the optimizer and the set of multidisciplinary analyses. The remaining communication requirements involved coordination among the analyses. Similarly, in the allat-once strategy, 40 variables were shared between the optimizer and the analyses, while 25 variables were passed among the analyses. In contrast, the collaborative architecture only requires communication of 23 variables between the system-level and the subspaces. This decreased level of communication is a direct result of empowering the subspaces with domain-specific decision responsibility.

In a multidisciplinary design environment, use of the collaborative architecture provides additional operational advantages as a result of its flexibility and modularity. Within this architecture, both analysis and optimization may be performed distributively. In fact, the distributed aspects of the architecture have been demonstrated on a set of heterogeneous computing platforms in Ref. [15]. Furthermore, in collaborative optimization, the required decomposition into a set of coordinated subspaces necessitates a coarse-grained modularity. This modularity allows the system to be easily extended and modified. Over the lifetime of a design project, this flexibility may be used to adjust the fidelity of the disciplinary models. In addition, changes to one domain-specific analysis may be altered without impacting the rest of the system. In contrast, the standard optimization approach provides very little flexibility and was found to be difficult to modify, while the all-at-once approach only provides a distributed analysis capability. 


\section{Summary}

In this investigation, the collaborative optimization architecture was used to perform multidisciplinary design of a dual-fuel, single-stage-to-orbit launch vehicle. Vehicle design, trajectory, and cost aspects were directly addressed. Posed to suit the collaborative architecture, this design problem was characterized by 95 design variables (23 interdisciplinary) and 16 constraints. A minimum development cost concept was obtained which compared favorably (within $0.1 \%$ in development cost) to a result produced by another optimization architecture. The influence of an a priori ascent-abort criterion on development cost and proper objective-function selection was discussed. Differences were highlighted between the minimum cost, weight, and $\Delta \mathrm{V}$ concepts.

The operational aspects of the collaborative architecture in a multidisciplinary design environment were presented and compared with two other optimization architectures. Relative to these other optimization strategies, the advantages of the collaborative architecture include no analysis integration requirements, the ability to use domain-specific analyses which already provide optimization without modification, inherent system flexibility and modularity, a distributed analysis and optimization capability, and a significant reduction in communication requirements. These practical advantages make the architecture well-suited for use in a large-scale, multidisciplinary design environment.

\section{References}

${ }^{1}$ Wurster, K., Rowell, L., and Peach, L., "The Next Generation Manned Launch System - A Complex Decision." AIAA Paper 93-4160, Sept. 1993.

${ }^{2}$ Freeman, D., Wilhite, A., and Talay, T., "Advanced Manned Launch System Study Status." IAF Paper 91-193, Oct. 1991.

${ }^{3}$ Stanley, D., Talay, T., Lepsch, R., Morris, W., and Wurster, K., "Conceptual Design of a Fully Reusable Manned Launch System," Journal of Spacecraft \& Rockets, Vol. 29, No. 4, July-Aug. 1992, pp. 529-537.

${ }^{4}$ Stone, H., and Piland, W., "21st Century Space Transportation System Design Approach: HL-20 Personnel Launch System," Journal of Spacecraft \& Rockets, Vol. 30, No. 5, Sept.-Oct. 1993, pp. 521528.

${ }^{5}$ Wilhite, A., Bush, L., Cruz, C., Lepsch, R., Morris, W., Stanley, D., and Wurster, K., "Advanced Technologies for Rocket Single-Stage-to-
Orbit Vehicles," Journal of Spacecraft \& Rockets, Vol. 28, No. 6, Nov.-Dec. 1991, pp. 646-651.

${ }^{6}$ Stanley, D., and Piland, W., "Technology Requirements for Affordable Single-Stage Rocket Launch Vehicles," Space Technology, Vol. 14, No. 5, 1994, pp. 319-330.

${ }^{7}$ Freeman, D., Stanley, D., Camarda, C., Lepsch, R., and Cook, S., "Single-Stage-To-Orbit: A Step Closer." IAF Paper 94-V3.534, Oct. 1994.

${ }^{8}$ Eldred, C., Powell, R., and Stanley, D., "Single Stage Rocket Options for Future Launch Vehicles." AIAA Paper 93-4162, Sept. 1993.

${ }^{9}$ Stanley, D., et al., "Rocket-Powered SingleStage Vehicle Configuration Selection and Design." AIAA Paper 93-1053, Feb. 1993.

${ }^{10}$ Freeman, D., Talay, T., and Austin, R., "Single-Stage-To-Orbit: Meeting the Challenge," IAF Paper 95-V.5.07, Oct. 1995.

11 "Reusable Launch Vehicle: Technology Development and Test Program." National Research Council, National Academy Press, 1995.

${ }^{12}$ Sobieszczanski-Sobieski, J., and Haftka, R., "Multidisciplinary Aerospace Design Optimization: Survey of Recent Developments." AIAA Paper 960711, Jan. 1996.

${ }^{13}$ Cramer, E., Dennis, Jr., J., Frank, P., Lewis, R., and Shubin, G., "Problem Formulation For Multidisciplinary Optimization," SIAM Journal of $O p$ timization, Vol. 4, No. 4, Nov. 1994, pp. 754-776.

${ }^{14}$ Braun, R., Collaborative Optimization: An Architecture for Large-Scale Distributed Design. PhD thesis, Stanford University, June 1996.

${ }^{15}$ Kroo, I., Altus, S., Braun, R., Gage, P., and Sobieski, I., "Multidisciplinary Optimization Methods for Aircraft Preliminary Design." AIAA Paper 94-4325, Sept. 1994.

${ }^{16}$ Braun, R., and Kroo, I., "Development and Application of the Collaborative Optimization Architecture in a Multidisciplinary Design Environment," in Multidisciplinary Design Optimization: State of the Art, SIA M, 1996.

${ }^{17}$ Kroo, I., "Decomposition and Collaborative Optimization for Large-Scale Aerospace Design," in Multidisciplinary Design Optimization: State of the Art, SIAM, 1996.

${ }^{18}$ Gage, P., Braun, R., Sobieski, I., and Kroo, I., "Collaborative Optimization: Convergence and Performance." AIAA Paper 96-4017, Sept. 1996.

${ }^{19}$ Sobieski, I., and Kroo, I., "Collaborative Optimization Applied to an Aircraft Design Problem," AIAA Paper 96-0715, Jan. 1996.

${ }^{20}$ Engelund, W., Stanley, D., McMillin, M., and Unal, R., "Aerodynamic Configuration Design Us- 
ing Response Surface Methodology Analysis." AIAA Paper 93-3967, Aug. 1993.

${ }^{21}$ Lepsch, R., Stanley, D., and Unal, R., "DualFuel Propulsion in Single-Stage Advanced Manned Launch System Vehicle," Journal of Spacecraft 86 Rockets, Vol. 32, No. 3, May-June 1995, pp. 417425 .

${ }^{22}$ Moore, A., Braun, R., and Powell, R., "The Infusion of Cost Into the Multidisciplinary Design of Space Transportation Systems," in The International Society of Parametric Analysts, 17th Annual Conference, June 1995.

${ }^{23}$ Moore, A., Braun, R., and Powell, R., "Optimal Technology Investment Strategies for a Reusable Launch Vehicle." AIAA Paper 96-4091, Sept. 1996.

24 “Access to Space Study Advanced Technology Team Final Report, Volume 2: Technology Plan," July 1993.

${ }^{25}$ Brauer, G., Cornick, D., and Stevenson, R., "Capabilities and Applications of the Program to Optimize Simulated Trajectories," NASA CR 2770 , Martin Marietta Corporation, Feb. 1977.

26 "PRICE-H Reference Manual," 1st edition, Martin Marietta PRICE (TM) Systems, Nov. 1993.

${ }^{27}$ Freeman, D., et al., "Future Space Transportation System Study." Aerospace America, pp. 36-56, June 1983.

${ }^{28}$ Martin, J., et al., "Orbit on Demand." Aerospace America, pp. 36-61, Feb. 1985.

${ }^{29}$ Olds, J., Multidisciplinary Design Techniques Applied to Conceptual Aerospace Vehicle Design. PhD thesis, North Carolina State University, 1993.

${ }^{30}$ Unal, R., Stanley, D., Engelund, W., and Lepsch, R., "Design for Quality Using Response Surface Methods: An Alternative to Taguchi's Parameter Design Approach," Engineering Management Journal, Vol. 6, No. 3, Sept. 1994.

${ }^{31} \mathrm{Olds}$, J., "System Sensitivity Analysis Applied to the Conceptual Design of a Dual-Fuel Rocket SSTO." AIAA Paper 94-4339, Sept. 1994.

${ }^{32}$ Nguyen, H., "FONSIZE: A Trajectory Optimization and Vehicle Sizing Program," AIA A Paper 93-1100, AIAA/AHS/ASEE Aerospace Design Conference, Irvine, CA, Feb. 1993.

${ }^{33}$ Pribnow, R., "Launch Vehicle Synthesis Methodology," SAWE Paper 1992, May 1991.

${ }^{34}$ Eldred, C., and Gordon, S., "A Rapid Method for Optimization of the Rocket Propulsion System for Single-Stage-to-Orbit Vehicles." NASA TN D8078 , July 1976.

${ }^{35}$ Wilhite, A., "Optimum Wing Sizing of a SingleStage-to-Orbit Vehicle," Journal of Spacecraft \&6 Rockets, Vol. 20, No. 2, Mar.-Apr. 1983, pp. 115-121.
${ }^{36}$ Braun, R., Powell, R., Lepsch, R., Stanley, D., and Kroo, I., "Comparison of Two Multidisciplinary Optimization Strategies for Launch Vehicle Design," Journal of Spacecraft 8 Rockets, Vol. 32, No. 2, Mar.-Apr. 1995, pp. 404-410.

${ }^{37}$ Gage, P., New Approaches to Optimization in Aerospace Conceptual Design. PhD thesis, Stanford University, 1995.

${ }^{38}$ Braun, R., Gage, P., and Kroo, I., "PostOptimality Analysis in Aerospace Vehicle Design." AIAA Paper 93-3932, Aug. 1993.

${ }^{39}$ Gill, P., Murray, W., Saunders, M., and Wright, M., "User's Guide for NPSOL (Version 4.0): A Fortran Package for Nonlinear Programming," Technical Report SOL 86-2, Dept. of Operations Research, Stanford University, Jan. 1986.

${ }^{40}$ Naftel, C., Lepsch, R., Powell, R., and Bacon, J., "Ascent Abort Capability for a Rocket-Powered Single Stage Advanced Manned Launch System." AIAA Paper 93-3694, Aug. 1993. 